\title{
Presencia de enfermedad coronaria obstructiva en pacientes con SPECT negativo sometidos a coronario- grafía por tomógrafo de 64 canales
}

Drs. César Parra $B^{(1)}$, Fabricio Sasia $T^{(1)}$, Lucrecia Burgos $E^{(1)}$, Leandro Lasave $E^{(1,2)}$, Stella Maris Batalles $V^{(2),}$ Eduardo Picabea $P^{(1,2)}$, Roberto Villavicencio $C^{(1)}$.

1. Servicio de Radiología. Diagnóstico Médico Oroño. Fundación Dr. J. R. Villavicencio. Rosario. Argentina.

2. Servicio de Hemodinamia y Cardiología Intervencionista. Instituto Cardiovascular de Rosario. Argentina.

Obstructive coronary artery disease in patients with negative SPECT undergoing coronary angiography with a 64-slice CT angiogram

Abtract: Objective. To evaluate the presence of obstructive coronary artery disease $(O C D)$ in patients with negative SPECT undergoing 64-slice coronary CT angiography. Material and methods. From July 2007 to January 2010, patients with negative SPECT undergoing CT angiography were assessed. We used a Philips Brilliance 64-slice CT scanner and low osmolality iodinated contrast. Coronary arteries were divided into 16 segments and the presence of OCD (lesion> 50\%), non-obstructive coronary disease (lesions $<50 \%$ ), or absence of coronary disease were evaluated. Results. From a population of 447 patients undergoing coronary angiography, 52 patients with negative SPECT were included in the study. The mean age was 60 years, and $67 \%$ corresponded to female patients. We observed 13 patients (25\%) with OCD. Percentages of arterial anomalies were as follows: $92 \%$, anterior descending artery; $53.8 \%$, right coronary artery; $46.1 \%$, circumflex coronary artery; $23 \%$, lateroventricular coronary artery; $15.3 \%$, posterior descending coronary artery; $7.7 \%$, diagonal coronary artery. No lesion was found in the left coronary artery. Conclusion In our analysis, $25 \%$ of patients with negative SPECT showed significant obstructive lesions.

Key Words: Coronary CT, Coronary obstruction, Ischemic heart disease.

Resumen: Objetivo. Evaluar la presencia de enfermedad coronaria obstructiva (ECO) en pacientes con SPECT negativo sometidos a Coronariografía TC64. Material y métodos. Se evaluaron pacientes sometidos a coronariografía TC con SPECT negativo desde julio 2007 a enero 2010.Se utilizó un tomógrafo Philips Brilliance 64 y contraste iodado de baja osmolaridad. Las arterias coronarias se dividieron en 16 segmentos evaluando la presencia de ECO (lesión > 50\%), enfermedad coronaria no obstructiva (lesión <50\%) o sin enfermedad coronaria. Resultados. Del total de 447 pacientes, se incluyeron 52 pacientes con SPECT negativo. La edad media fue 60 años, $67 \%$ mujeres. Se observaron 13 pacientes (25\%) con ECO. Las afecciones arteriales fueron: arteria descendente anterior en el $92 \%$, coronaria derecha $53,8 \%$, circunfleja $46,1 \%$, lateroventricular $23 \%$,descendente posterior $15,3 \%$, diagonal $7,7 \%$ y ninguna en el tronco coronario izquierdo. Conclusión. Según nuestra serie, el 25\% de los pacientes con SPECT negativo presentó lesiones obstructivas significativas.

Palabras clave: Cardiopatía isquémica, Obstrucción coronaria, Tomografía coronaria.

Parra C. Presencia de enfermedad coronaria obstructiva en pacientes con SPECT negativo sometidos a coronariografía por tomógrafo de 64 canales Rev Chil Radiol 2011; 17(2): 77-79.

Correspondencia a: Dr. César Parra B. / matiasparra@gmail.com

Trabajo premiado en categoría oral, Sección Cardiovascular, durante el XXV Congreso Interamericano de Radiología, efectuado en Santiago de Chile.

\section{Introducción}

La tomografía computarizada con emisión de fotón único (SPECT) es el método no invasivo más utilizado en el diagnóstico de enfermedad arterial coronaria obstructiva (ECO) y aunque su valor predictivo negativo es limitado ${ }^{(1-3)}$, habitualmente los pacientes con SPECT negativo no son sometidos a estudios más invasivos. La coronariografía por tomógrafo de 64 canales (TC64) ha demostrado tener un alto valor predictivo negativo y una excelente correlación con la coronariografía invasiva ${ }^{(4,5)}$.
El objetivo de este estudio fue evaluar la presencia de enfermedad coronaria obstructiva en aquellos pacientes con SPECT negativo, que posteriormente fueron sometidos a coronariografía TC64.

\section{Material y métodos}

Se evaluaron pacientes sometidos a coronariografía TC64 desde julio de 2007 a enero de 2010.

Se incluyeron sólo aquellos con estudio de perfusión miocárdica negativo. Los pacientes fueron 
sometidos a TC64 por indicación primaria de su médico de cabecera debido a la interpretación de la clínica y estudio funcional.

Se excluyeron los pacientes con síndrome coronario agudo, cirugía de revascularización miocárdica previa, score de calcio mayor a 1.000 y aquellos pacientes con antecedentes de reacciones alérgicas al contraste iodado. Se utilizó un tomógrafo Philips Brilliance 64 , sincronizado con electrocardiograma, de donde retrospectivamente se utilizaron los datos correspondientes al $75 \%$ del intervalo R-R.

Se utilizó una bomba de infusión de doble cabezal (Medrad Stellant ${ }^{\circledR}$ ), con contraste iodado de baja osmolaridad (Optiray ${ }^{\circledR}$-loversol-350), inyectado por una vena antecubital derecha, en un bolo de 80 a $100 \mathrm{ml}$, seguido de una infusión de $40 \mathrm{ml}$ de solución salina. Se ubicó el sensor de contraste a nivel de la aorta ascendente y la adquisición se inició 5 segundos después que la atenuación llegó a $120 \mathrm{UH}$.

Antes de la realización del procedimiento, se evaluó la frecuencia cardíaca (FC). En aquellos pacientes con FC mayor a 68 lat/min se administró metoprolol IV hasta una dosis de $15 \mathrm{mg}$ según la respuesta. Ningún estudio se realizó con FC mayor a 68 lat $/ \mathrm{min}$. En los pacientes con $\mathrm{FC}$ basal mayor a 80 lat/min se optimizó el tratamiento oral con betabloqueantes, antagonistas cálcicos o ivabradina según el criterio del médico tratante y siendo reevaluado una semana después.

Para la interpretación de los estudios se utilizó una estación de trabajo Philips Brillance 190P utilizando el software Complex Cardiac. Se analizaron los cortes axiales, reconstrucciones multiplanares y cortes finos en proyección de máxima intensidad (MIP).

Las arterias coronarias se dividieron en 16 segmentos siendo visualizados por lo menos en dos planos, evaluando la presencia de ECO (lesión $>50 \%$ ), enfermedad coronaria no obstructiva (lesión < 50\%) o la ausencia de enfermedad coronaria.

Las variables cuantitativas se presentan como media y desvío estándar, o mediana y percentilo (25$75)$ según corresponda. Las variables categóricas se muestran como números y porcentajes.

\section{Resultados}

Del total de 447 pacientes sometidos a coronariografía, se incluyeron 52 pacientes con SPECT negativo.

La edad media fue $61.9 \pm 11.1$ años, constituido por un $67 \%$ de mujeres.

El $65.3 \%$ de los pacientes eran hipertensos (HTA), el $57.6 \%$ dislipémicos (DLP), el $3.8 \%$ diabéticos (DBT) y el $30.7 \%$ eran tabaquistas (TBQ). La media del score de calcio para estos pacientes fue de $212 \pm 321.9$ (Tabla I). A través de la TC64 se observaron 14 pacientes (26.9\%) sin lesiones coronarias (Figura 1), 25 (48\%) con lesión coronaria menor al 50\% (Figura 2) y 13 pacientes $(25 \%)$ con enfermedad coronaria obstructiva (Figura 3) (Tabla II).
Tabla I. Característica basal de los pacientes.

52 pacientes - 832 segmentos arteriales

\begin{tabular}{lr} 
Edad & $61.9 \pm 11.1 \mathrm{años}$ \\
Mujeres & $67.0 \%$ \\
HTA & $65.3 \%$ \\
DLP & $57.6 \%$ \\
DBT & $3.8 \%$ \\
Tabaquismo & $30.7 \%$ \\
PAS & $125 \pm 9.2 \mathrm{mmHg}$ \\
PAD & $73 \pm 6.5 \mathrm{mmHg}$ \\
Administración BBEV & $69.2 \%$ \\
FC inicio & $60.1 \pm 3.4 \mathrm{lat} / \mathrm{min}$ \\
Score de Calcio & $212 \pm 321$ \\
\multicolumn{2}{l}{} \\
HTA: Hipertensión arterial, DLP: dislipemia, DBT: diabetes \\
BBEV: Beta bloqueantes endovenosos.
\end{tabular}

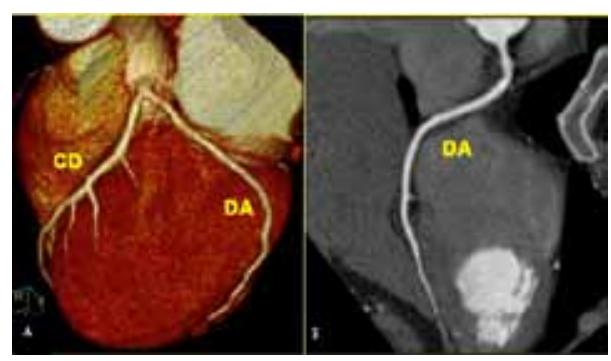

Figura 1. Estudio de coronariografía CT64 normal. A: Reconstrucción volumétrica. B: Reconstrucción multiplanar MIP. CD: Art. Coronaria derecha. DA: Art. Descendente anterior.

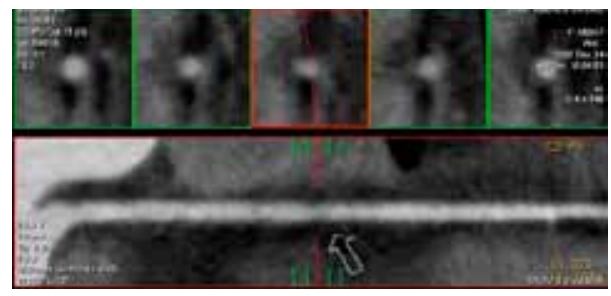

Figura 2. Estenosis no obstructiva en arteria coronaria derecha (Flecha). Reconstrucción multiplanar.

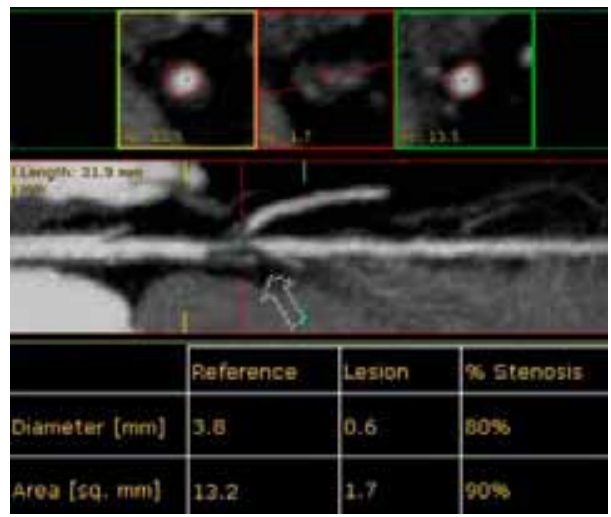

Figura 3. Estenosis obstructiva mayor al $50 \%$ en arteria descendente anterior (Flecha). Reconstrucción multiplanar. 
Tabla II. Hallazgos en angiografía TC64 en pacientes con SPECT negativo.
Lesión menor al $50 \%$

Sin lesión
Lesión mayor $50 \%$
$13(25 \%)$

25 (48\%)
$14(26.9 \%)$

La arteria mayormente afectada fue la arteria descendente anterior, encontrándose lesión en 12 pacientes (92\%) (Figura 4), luego la coronaria derecha en $7(53,8 \%)$, la circunfleja en $6(46,1 \%)$, la lateroventricular en $3(23 \%)$, la descendente posterior en 2 $(15,3 \%)$, la diagonal en un paciente $(7,7 \%)$ y ninguna alteración del tronco coronario izquierdo (Tabla III).

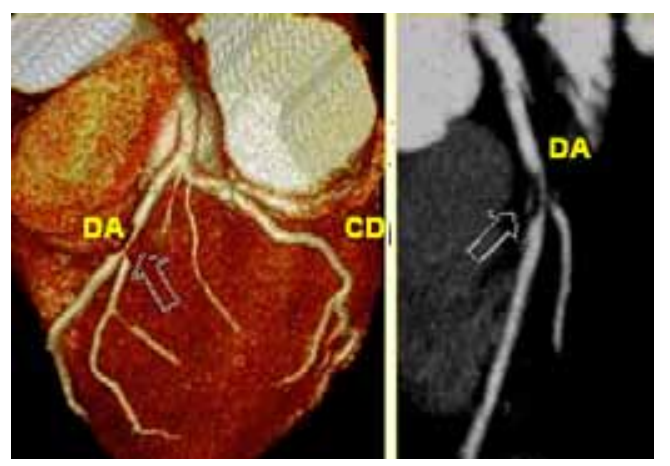

Figura 4. Estenosis obstructiva en la arteria descendente anterior (Flecha). A: Reconstrucción volumétrica. B: Reconstrucción multiplanar MIP. DA: a. descendente anterior. CD: a. coronaria derecha.

Tabla III. Distribución de las lesiones en el árbol coronario.

\section{Pacientes con TCMS $>50 \% 122$ segmentos afectados}

Descendente anterior
Coronaria derecha
Circunfleja
Latero ventricular
Descendente posterior
Diagonal
T. Coronario izquierdo

\section{Discusión}

En este tipo de pacientes, con sospecha de enfermedad coronaria y SPECT negativo evaluados mediante TC64 observamos presencia de lesión obstructiva en el $25 \%$ de los pacientes siendo la arteria descendente anterior el vaso más afectado. Estos resultados se encuentran en concordancia con datos de dos estudios recientes. En el primero (Van Werkhoven et al) se evaluaron 274 pacientes con SPECT negativo y el $22 \%$ presentó lesiones coronarias significativas por TC64(6). En el segundo estudio, de Schuijf et al, se observó un
$55 \%$ de lesiones coronarias en pacientes con estudios de perfusión normal ${ }^{(1)}$.

Diferentes estudios demostraron que en pacientes con sospecha de enfermedad coronaria, la evaluación mediante SPECT puede ser negativa aun en presencia de aterosclerosis subclínica, que puede estar asociado a un alto riesgo de eventos cardíacos a largo plazo ${ }^{(7,8)}$. Por su parte la TC64, a pesar de que podría sobreestimar las estenosis (en especial cuando hay importante calcificación) en relación a la coronariografía convencional, provee información adicional de la morfología de la placa que puede ser útil en la toma de decisiones terapéuticas ${ }^{(1,4)}$.

De esta manera, la utilización de la TC64 para evaluación de los pacientes con sospecha de coronariopatía y estudios de inducción de isquemia negativos o no concluyentes colabora de manera importante en la caracterización real de la enfermedad coronaria, permitiendo el tratamiento adecuado en intensivo en aquellos pacientes con enfermedad obstructiva y la prevención primaria en aquellos pacientes con enfermedad coronaria no obstructiva.

\section{Bibliografía}

1. Schuijf JD, Wijns W, Jukema JW, et al. Relationship between noninvasive coronary angiography with multi-slice computed tomography and myocardial perfusion imaging. J Am Coll Cardiol 2006; 48: 2508-2514.

2. Metz LD, Beattie M, Hom R, Redberg RF, Grady D, Fleischmann KE. The prognostic value of normal exercise myocardial perfusion imaging and exercise echocardiography: a meta-analysis. J Am Coll Cardiol 2007; 49: 227-237.

3. Germano G, Kavanagh PB, Waechter P, et al. A new algorithm for the quantitation of myocardial perfusion SPECT. I: technical principles and reproducibility. J Nucl Med 2000; 41: 712-719.

4. Gaemperli O, Schepis T, Koepfli P, et al. Accuracy of 64-slice CT angiography for the detection of functionally relevant coronary stenoses as assessed with myocardial perfusion SPECT. Eur J Nucl Med Mol Imaging 2007; 34 : 1162-71.

5. Elhendy A, Schinkel A, Bax J, van Domburg T, Poldermans D. Long-term prognosis after a normal exercise stress Tc-99m sestamibi SPECT study. J Nucl Cardiol 2003; 10: 261-266.

6. Van Werkhoven J, Schuijf J, Gaemperli O, Wouter Jukema J, Boersma E, Wijns W, et al. Prognostic Value of Multislice Computed Tomography and Gated Single-Photon Emission Computed Tomography in Patients With Suspected Coronary Artery Disease. J Am Coll Cardiol 2009; 53; 623-632.

7. Gaemperli O, Schepis T, Valenta I, Koepfli P, Husmann $\mathrm{L}$, Scheffel $\mathrm{H}$, et al. Functionally relevant coronary artery disease: comparison of 64-section CT angiography with myocardial perfusion SPECT. Radiology 2008; 248(2): 414-423.

8. Berman DS, Hachamovitch R, Shaw LJ, Friedman JD, Hayes SW, Thomson LE, et al. Roles of nuclear cardiology, cardiac computed tomography, and cardiac magnetic resonance: noninvasive risk stratification and a conceptual framework for the selection of noninvasive imaging tests in patients with known or suspected coronary artery disease. J Nucl Med 2006; 47: 1107-1118. 